\title{
Prescription auditing based on World Health Organization (WHO) prescribing indicators in a teaching hospital in North India
}

\author{
Ahsan M. ${ }^{1}$, Shaifali I. ${ }^{2}$, Mallick A.K. ${ }^{3}$, Singh H.K. ${ }^{4}$, Verma S. ${ }^{5}$, Shekhar A. ${ }^{6}$ \\ ${ }^{1}$ Dr. Marya Ahsan, Assistant Professor, ${ }^{2}$ Dr Iram Shaifali, Assistant Professor, ${ }^{3}$ Dr Ayaz Khurram Mallick, Associate \\ Professor, Department of Biochemistry, ${ }^{4}$ Dr Hari Om Kumar Singh, Professor, ${ }^{5}$ Dr Swetabh Verma, Post graduate \\ student, ${ }^{6}$ Dr Amritanshu Shekhar, Post graduate student, all authors are affiliated with Department of Pharmacology, \\ Rohilkhand Medical College \& Hospital, Bareilly, UP, India.
}

Address for Correspondence: Dr. Marya Ahsan, Assistant Professor, Department of Pharmacology, Rohilkhand Medical College, Bareilly, Uttar Pradesh. Email: marya_ahsan@yahoo.co.in

\begin{abstract}
Background: Prescription writing is an important means of therapeutic intervention by the doctor and reflects his approach towards safe prescribing. Complacency in prescribing results in errors which can even cause adverse effects. These errors can be detected through a prescription audit. We undertook this study to audit the out-patient department (OPD) prescriptions for completeness of prescription format, legibility and against the World Health Organization (WHO) prescribing indicators recommended to investigate rational use of drugs. Methods: OPD prescriptions were photographed from the pharmacy of a teaching hospital over a period of three months and 1274 prescriptions were audited. Prescriptions were evaluated for completeness of prescription format while legibility was graded as grade 1,2 and 3. Prescriptions were also analyzed on the five WHO prescribing indicators. Results: An average of $4.02 \pm 2.23$ drugs were prescribed per prescription of which $39.01 \%$ were antibiotics. Though $79.2 \%$ drugs were prescribed from the Essential Drug List, none was prescribed by the generic name. Rate of injection use was $7.54 \%$ in our study. Registration number of the doctor was absent in $100 \%$ of the prescriptions. Errors such as omitting the mention of allergy status, follow-up advice and directions of use were common. Almost $8 \%$ prescriptions were illegible (grade 3) and 66.8\% were legible with difficulty (grade 2). Conclusion: Majority of the prescriptions were incomplete and poly-pharmacy was evident in our study. Regular auditing and feedback is necessary for imbibing safe prescribing practices. Doctors need to be made aware about the errors and the recommended guidelines.
\end{abstract}

Key words: Auditing, Prescription format, legibility, Prescribing indicators

\section{Introduction}

Writing a prescription is an important mode of therapeutic intervention by the doctor for the patient. Prescription writing is a skill acquired through training. The quality of a prescription reflects the competence of a physician and his attitude towards rational prescribing. However, systematic reviews suggest that prescribing errors are common and can affect from 4.2 to $82 \%$ of prescriptions [1]. These prescribing errors can also cause adverse effects. Almost four in 1000 prescriptions have errors that have the potential for causing adverse effects [2]. Error can arise from any

Manuscript received $24^{\text {th }}$ September 2016

Reviewed: $6^{\text {th }}$ October 2016

Author Corrected: $18^{\text {th }}$ October 2016

Accepted for Publication $31^{\text {st }}$ October 2016 step of prescribing such as the choice of drug, dose, route of administration and wrong frequency or duration of treatment. Inaccuracy in writing and poor legibility of handwriting or incomplete writing of a prescription can lead to misinterpretation, thus leading to errors in dispensing and administration.

Both individual and system related factors are responsible for prescribing errors [3]. Detection is the first crucial step in building safer systems and preventing errors. These errors can be detected by systematic analysis of prescriptions through a prescription audit [4]. Audit was first used by Florence Nightingale in 1854 to prevent post surgical mortality. 
Prescription audit is part of the holistic clinical audit which was defined in a paper Principles for Best Practice in Clinical Audit as "a quality improvement process that seeks to improve patient care and outcomes through systematic review of care against explicit criteria and the implementation of change" [5]. US philosopher W. Edwards Deming, had characterized the audit framework as a sequence of events, the Deming cycle (Plan-Do-Check-Act).

Audit is a simple tool to measure and monitor what we do against a reference standard. Prescription auditing is also an educational activity, and if regularly done can aid in improving the prescription quality and thus enable the patient to receive high standard and best quality care [5].

We undertook the study to audit the quality of outpatient department prescriptions at our hospital for completeness, legibility and against the World Health Organization (WHO) recommended core prescribing indicators to investigate rational use of drugs [6].

\section{Materials and Methods}

The study was designed and conducted as a prospective observational study at a teaching hospital in North India after taking ethical clearance from the Institutional Ethics Committee.

The study was carried over a period of three months from December 2015 to February 2016. A total of 1274 out-patient prescriptions were randomly sampled and photographed from the hospital pharmacy with a digital camera irrespective of patient characteristics, diagnosis and hospital department.

The details of all the prescriptions were analyzed on the following parameters:

- Prescription format and its completeness with regards to:

\section{Results}

Among the 1274 prescriptions analyzed, all of them had the date, details of the patient such as name, age, sex and address. Weight was written on all pediatric prescriptions but not on prescriptions for adults. Name of all the unit doctors and hospital address was printed on the prescriptions, but none mentioned the doctor's registration number and $17 \%$ prescriptions did not have the physician's initials. Complete diagnosis was written in only $56 \%$ of prescriptions. In the inscription part of the prescription, the dosage form such as Tab, Inj was missing in $15 \%$. Nine percent of prescriptions had incorrect dosage and $13 \%$ of prescriptions omitted the duration of treatment (figure 1). Direction for drug use was not mentioned in $35 \%$ of prescriptions while follow up advice was written in only $23 \%$ of prescriptions. $\checkmark$ patient identifications (name, age, sex, weight, address)

$\checkmark$ prescriber identification (name, department, hospital, registration number, physician initials).

$\checkmark$ writing (start date, strength / dose / product formulation)

$\checkmark$ dosing (under-dosing and overdosing)

$\checkmark$ duration of treatment

$\checkmark$ directions for administration

$\checkmark$ follow up advice

$\checkmark$ allergy status

$\checkmark$ diagnosis

- Legibility of prescriptions: Prescription legibility was graded on a subjective scale by two independent investigators. Prescriptions were graded as:

$\checkmark$ Grade 1 (legible with ease)

$\checkmark$ Grade 2 (legible with difficulty)

$\checkmark$ Grade 3 (illegible).

In case of discrepancy between the grading of two investigators, opinion of a third investigator was sought.

- WHO core prescribing indicators which includes [6].

Average number of drugs per prescription - Fixed dose combinations were also counted as one drug

$\checkmark$ Percentage of drugs prescribed by generic name antibiotics were classified based on the WHO model list for antibiotic classification and included penicillin, other antibiotics, anti-infective dermatological drugs, anti-infective ophthalmological agents and anti-diarrhoeal drugs or their combinations

$\checkmark$ Percentage of injections per prescription vaccinations were excluded from this list

$\checkmark$ Percentage of drugs prescribed from the Essential Drugs List. $\checkmark$ Percentage of antibiotics per prescription- 


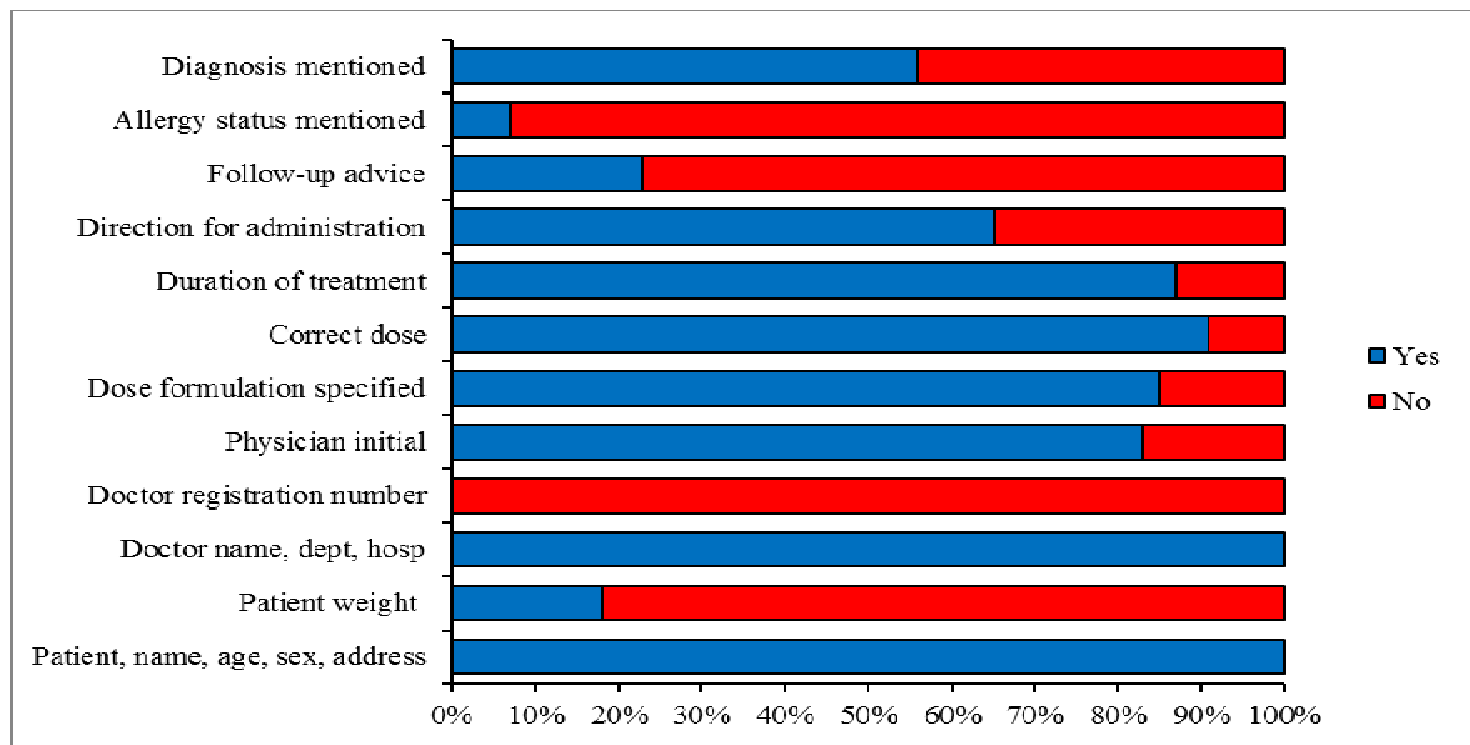

Figure-1:A bar graph showing completeness of prescription

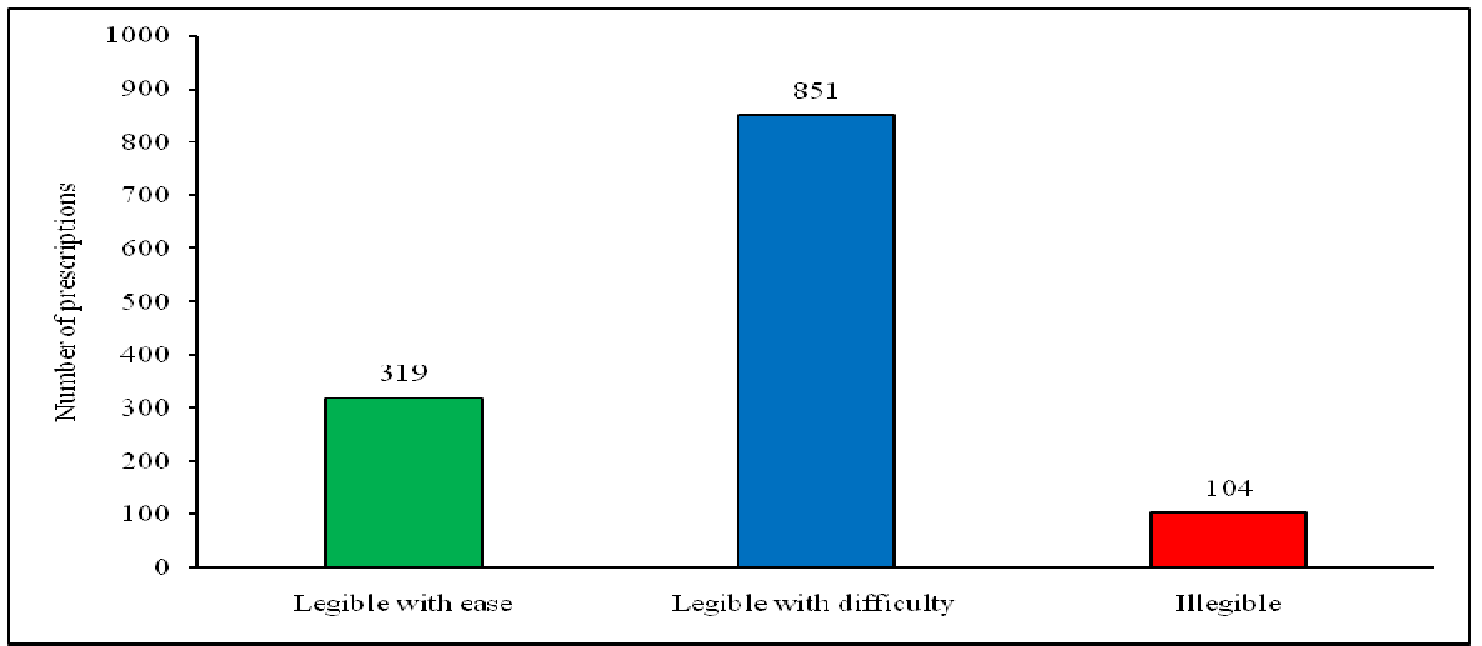

Figure-2: A bar graph showing level of legibility of prescriptions and its frequency.

As seen in figure 2, 25.03\% of the prescriptions were legible with ease while $66.8 \%$ of prescriptions were legible with difficulty and $8.16 \%$ were illegible. Capital letters were not used in any of the prescriptions. A total of 5123 drugs were prescribed in 1274 prescriptions. The average number of drugs per prescription was $4.02 \pm 2.23$ and ranged from one to eight drugs. While 1009 drugs were prescribed from the EDL, not a single drug was prescribed by the generic name. Among the total drugs, 498 were antibiotics and 96 were injections (Table 1).

Table-1: A table showing the data based on WHO prescribing indicators.

\begin{tabular}{|c|c|c|}
\hline WHO core prescribing indicators & Total number of drugs & $\begin{array}{c}\text { Average/Percentage per prescription } \\
(\mathbf{n}=\mathbf{1 2 7 4})\end{array}$ \\
\hline Drugs prescribed & 5123 & 4.02 \\
\hline Drugs prescribed by generic name & 0 & 0 \\
\hline Antibiotics & 498 & 7.54 \\
\hline Injections & 96 & 79.2 \\
\hline Drugs from EDL & 1009 & 01 \\
\hline
\end{tabular}




\section{Discussion}

Prescription is an important intervention by the physician and it is the ethical and legal duty of the practitioner to write complete and legible prescriptions. In our study we found that the date of prescription and patient's details (name, age, sex, and address) were complete in all the prescriptions. This was because these details were printed at the time of registration itself. Studies auditing hand written prescriptions have found that patient details were usually incomplete in almost all prescriptions [7]. Patient details are important for ensuring that the correct patient receives the medicines and also for medico-legal and record-keeping purposes. Also while prescribing drugs with contraindications or for special populations, it is very important to have the gender, age and weight of the patient. Weight of the patient was mentioned in $17 \%$ of the prescriptions in our study but these prescriptions mostly belonged to the pediatric department.

Though the department and unit details were printed on all the prescriptions, the name of the prescribing doctor was not evident in $17 \%$ prescriptions as they did not have the physician initials. Prescriptions, in which the name of the prescribing doctor is not clear, invalidate the prescription and can cause inconvenience to the patient as some drugs won't be dispensed by the pharmacist in such cases. Physician initials also help in identifying the prescribing doctor in an entire unit where interns, junior and senior residents and unit heads work together. Moreover, the registration number of the doctors was not mentioned on any of the prescriptions.

On analyzing the prescriptions we found that the prescriptions were incomplete with regards to mention of allergy status, follow-up advice and direction of administration. This was followed in frequency by writing errors such as omitting mention of drug formulation. Dosing errors and omitting duration of treatment was also common prescribing errors in $9 \%$ and $13 \%$ prescriptions respectively (figure1). Our findings were in line with studies done in other parts of India but much higher rate of error was found in comparison to other global studies [8,9]. Most drugs are available in variable strengths and dosage forms and thus it poses problems for dispensing. It can also lead to issues such as treatment failure, antibiotic resistance and toxicities which are associated with under-dosing or overdosing. Wrong dose, dose omission, and wrong time were the most common type of prescribing errors found in many studies worldwide $[9,10]$. Our study also highlighted that specific directions to patient regarding drug use were missing in $65 \%$ and follow up advice was omitted in $77 \%$ of prescriptions. This could be because the doctor's tend to rely on verbal communication rather than writing it down. It could also be an outcome of heavy OPD load. Prescriptions incomplete with regards to allergy status of the patient open up a window for adverse drug reaction especially if drug to which the patient is allergic is prescribed. Though the WHO does not stress on writing the diagnosis on the prescription, it can help in clearing doubts during dispensing if drugs with similar names are prescribed.

A large number of medication errors have been blamed on illegible writing of the prescriber. Illegible writing creates ambiguity and can potentially lead to dispensing of wrong drugs which can result in serious adverse events and even death [10]. In our study, $8.16 \%$ of the prescriptions were illegible and $66.8 \%$ were legible only with difficulty (figure 2). To avoid such confusions, regulatory bodies in India advocate the use of capital letters while prescribing drugs [11]. Moreover, places where electronic prescriptions are used, the rate of such errors are negligible [12]. In advanced healthcare setups using the electronic prescribing systems, the rates of errors such as missing strength/dose, formulation not specified or no start date and errors due to legibility issues can be minimized significantly but they do not overcome the errors arising due to transcription mistakes. Electronic prescribing systems are themselves associated with a new pattern of errors [9].

To investigate the drug use in health facilities, the WHO has recommended core prescribing indicators. These indicators aim to measure the performance of health care providers in several important areas pertaining to appropriate or rational use of drugs. These indicators have been developed by WHO after observing prescribing practices at outpatient facilities for the treatment of acute and chronic illnesses [6]. The average number of drugs per consultation in our study was 4.02 \pm 2.23 drugs (table 1) which was similar to drug use pattern in tertiary care hospitals (4.22) in other parts of India [8]. However, it was much higher than other studies done in secondary level hospitals (3.1) and rural India $(3.31)[13,14]$. It is an indicator of polypharmacy at our hospital. Polypharmacy increases the health care 
costs and has important implications on the national health budget. Polypharmacy also increases the risk of drug-drug interactions and adverse drug events. Since the study was done in a teaching hospital, most of the prescribing doctors hold a master's degree. Studies have shown that doctors with a higher degree prescribe significantly greater number of drugs than those with a bachelor's degree [15]. This highlights an important area of intervention where training prescribers for rational prescribing can help improve the quality of prescriptions and in attaining the WHO recommendation of 2.0 drugs per encounter [6]. Moreover, studies on predictors for prescribing errors concluded that the prescribing error risk increased by $14 \%$ for every additional drug prescribed [9]. Thus the single most important predictor for error is the number of drugs in a prescription.

All the drugs were prescribed by brand names in our study and not a single drug was prescribed by generic name. The reason for this is that the prescriber's try to adhere to the hospital formulary as the drugs are available at low rates in the hospital pharmacy. Also the doctors are skeptical about the efficacy and bioavailability of generic drugs. Generic prescribing should be encouraged as it reduces the chances of dispensing errors.

The percentage of antibiotics prescribed in our audit was $39.01 \%$ which is higher than the limits set by WHO $(20-25.4 \%)$. Though these rates were significantly higher than other studies done in tertiary setup $(17.48 \%)$, they were much lower than antibiotic use in private setup $(53.6 \%)$ and rural sector $(45 \%)$ [ $8,13,14]$. Whether the high prevalence of antibiotic use was inappropriate cannot be concluded as most of the patients attending the out-patient department are from rural background and frequently suffer from bacterial infections.

Percentage of injections in our study was only $7.54 \%$ which is well within the limits set by WHO $(\leq 10 \%)$. Oral route was the most commonly prescribed route in our study. It was comparable to other studies done in tertiary level hospital $(6.19 \%)$ [8]. The rate in our study was much lower than the injection encounters in private settings $(20.8 \%)$ or secondary level settings $(25 \%)$ $[7,13]$. The lower rate of injections in our study is encouraging as injections are associated with increased risk of blood-borne infections like HIV and hepatitis. In addition, non-sterile technique can also cause local irritation and sepsis. Lesser use also helps in limiting the health care cost especially when cheaper oral alternatives are available. $79.2 \%$ of the drugs were prescribed from the EDL or hospital formulary.

These rates are considerably higher than EDL prescribing frequency from other studies $[7,8,16]$. It points to the physician's inclination towards costeffective therapy. However, these rates were lower than reports from other developing countries where up to $99 \%$ of drugs are prescribed from the EDL [17]. Prescribers practices like prescribing from the EDL helps in promoting rational prescribing.

\section{Conclusion}

Our study revealed that prescribing errors were common in the out-patient department prescriptions at our hospital. In order to prevent these errors and reduce the risk of adverse reaction due to them, it is imperative that they be first detected systematically. Many prospective and retrospective methods can be used to detect and prevent the medication errors [4]. After the deficiencies have been highlighted, no audit is complete without suggesting solutions and planning its implementation.

Training and assessment of prescribers, regular monitoring, making prescriber's aware of the errors and the guidelines and open communication has been widely recommended as an important intervention tool to decrease the prevalence of prescribing errors. Studies have shown that if undergraduate students are given adequate training in safe and rational prescribing, the incidence of prescribing errors is significantly reduced [18]. In addition to this, there is a need to sensitize the prescribers for rational prescribing. As the study was done at a teaching hospital, most of the prescribing load is shared by junior and senior residents. Intervention in the form of training in rational prescribing at their level will help to facilitate the attainment of WHO targets for prescribing.

Funding: Nil, Conflict of interest: None initiated, Permission from IRB: Yes

\section{References}

1. Ross S, Bond C, Rothnie H, Thomas S, Macleod MJ. What is the scale of prescribing errors committed by junior doctors? A systematic review. $\mathrm{Br} \mathrm{J}$ Clin Pharmacol. 2009 Jun; 67 (6): 629-40. doi: 10.1111/j. 1365-2125. 2008.03330.x. Epub 2008 Oct 23. 
2. Velo GP, Minuz P. Medication errors: prescribing faults and prescription errors. Br $\mathrm{J}$ Clin Pharmacol 2009; 67(6): 624-8. doi: 10. 1111/j. 1365-2125. 2009. 03425.x.

3. Reason J. Human error: models and management. BMJ 2000 Mar 18;320(7237):768-70.

4. Montesi G, Lechi A. Prevention of medication errors: detection and audit. Br J Clin Pharmacol 2009; 67(6):651-655. doi:10.1111/j.1365-2125.2009. 03422.x.

5. National Institute for Clinical Excellence (NICE): Principles for Best Practice in Clinical Audit. Oxford: Radcliffe Publishing 2002.

6. World Health Organization:How to Investigate Drug Use in Health Facilities: Selected Drug Use Indicators EDM Research Series. http: // apps. who. int/ medicinedocs / en/d /Js2289e/ (accessed 26 september 2016).

7. Shelat PR, Kumbar SK. Analysis of Out Door Patients Prescriptions According to World Health Organization (WHO) Prescribing Indicators Among Private Hospitals in Western India. J Clin Diagn Res 2015; 9(3): FC01-4. doi: 10. 7860/JCDR/2015/ 12724. 5632.

8. Abidi A, Gupta S, Kansal S. Prescription auditing and drug utilization pattern in a tertiary care teaching hospital of western UP. Int J Basic Clin Pharmacol. 2012;1(3):184-190.doi:10.5455/2319-2003.ijbcp003812

9. Seden K, Kirkham JJ, Kennedy T. Cross-sectional study of prescribing errors in patients admitted to nine hospitals across North West England. BMJ Open 2013;3:e002036. doi:10.1136/bmjopen-2012- 002036.

10. Kiekkas P, Karga M, Lemonidou C, Aretha D, Karanikolas M. Medication errors in critically ill adults: A review of direct observation evidence. Am J Crit Care 2011;20(1):36-44. doi: 10.4037/ajcc2011331.
11. Model prescription format. http: // www. delhimedicalcouncil. Org / pdf / modalprescription. pdf (accessed 26 september 2016).

12. Albarrak AI, Al Rashidi EA, Fatani RK, Al Ageel SI, Mohammed R. Assessment of legibility and completeness of handwritten and electronic prescriptions. Saudi Pharm J. 2014 Dec; 22(6): 522-7. doi: 10. 1016 /j. jsps. 2014. 02. 013. Epub 2014 Mar 13.

13. Potharaju HR, Kabra SG. Prescription audit of outpatient attendees of secondary level government hospitals in Maharashtra. Indian J of Pharmacol. 2011; 43 (2):150-6. doi:10.4103/0253-7613.77350.

14. Patil KR, Mali RS, Dhangar BK, Bafna PS, Gagarani MB, Bari SB. Assessment of Prescribing Trends and Quality of handwritten Prescriptions from Rural India. Journal of Pharma Scitech 2015;5(1): 54-60.

15. Kun Y, Jianwen C, Hua F, Huifen C, Yizhong Z. Analysis of factors affecting physician's prescribing conduct. Chin J Hosp Admin 2002;18:92-4.

16. Hazra A, Tripathi SK, Alam MS. Prescribing and dispensing activities at the health facilities of a nongovernmental organization.Natl Med J India 2000 Jul-Aug;13:177-82.

17. El Mahalli AA. WHO/INRUD drug prescribing indicators at primary health care centres in Eastern province, Saudi Arabia. East Mediterr Health J Rev Santé Méditerranée Orient Al-Majallah Al-Ṣiḥhịyah LiSharq Al-Mutawassiṭ 2012;18(11):1091-6.

18. Agrawal A, Aronson JK, Britten N. Medication errors problems and recommendations from a consensus meeting. Br J Clin Pharmacol 2009 Jun;67(7):592-8. doi: $10.1111 / \mathrm{j} .1365-2125.2009 .03414 . \mathrm{x}$

\section{How to cite this article?}

Ahsan M, Shaifali I, Mallick A.K, Singh H.K, Verma S, Shekhar A. Prescription auditing based on World Health Organization (WHO) prescribing indicators in a teaching hospital in North India. Int J Med Res Rev 2016;4(10):18471852.doi:10.17511/ijmrr. 2016.i10.22. 